\title{
Cosmology, Particles, and the Unity of Science
}

\author{
Henrik Zinkernagel*
}

To appear in Studies in History and Philosophy of Modern Physics

\begin{abstract}
During the last three decades, there has been a growing realization among physicists and cosmologists that the relation between particle physics and cosmology may constitute yet another successful example of the unity of science. However, there are important conceptual problems in the unification of the two disciplines, e.g. in connection with the cosmological constant and the conjecture of inflation. The present article will outline some of these problems, and argue that the victory for the unity of science in the context of cosmology and particle physics is still far from obvious.
\end{abstract}

\section{Cosmology and Philosophy}

Given that cosmology has been part of human thought since the beginning of intellectual history, it is somewhat surprising that studies of the philosophy of scientific cosmology have been remarkably few. Scientific cosmology dates back at least to the beginning of this century, and although philosophers of science in the 20th century have seen the advent of the theories of relativity and quantum mechanics as the main events, the all-important role of cosmology in the shaping of a scientific 'world-view' has not been done justice by this limited philosophical attention. Perhaps this lack of interest has to do with the fact that cosmology, for a large part of this century, was not widely regarded as 'real' science. Indeed, as documented by Kragh [25], up to at least the late fifties there was no standard paradigm of cosmology, and more or less philosophical arguments flourished in the debate over steady-state theories vs. Big Bang alternatives. This situation changed drastically with the 1965 discovery of the cosmic background radiation, interpreted as the relic of the Big Bang, which firmly established what was to be regarded as 'normal science' cosmology.t

The 1965 event, however, has by no means removed the need for philosophical investigations of cosmology. In particular, the last thirty years of interaction between cosmology and particle physics have raised important questions regarding the relations between philosophy, physics and cosmology. During this epoch steps have been made towards the realization of a programme going back at least to Eddington in the early 1930s of an intimate connection between general relativity, the backbone theory of modern cosmology, and quantum mechanics [22]. Edward W. Kolb, a leading theoretical astrophysicist, has expressed the relation between cosmology and particle physics as follows:

\footnotetext{
${ }^{*}$ Consejo Superior Investigaciones Cientificas, Instituto de Filosofia, Calle Pinar 25, Madrid, Spain. e-mail: zink@ifs.csic.es

${ }^{1}$ A detailed account of the history and philosophy of cosmology before the microwave background radiation discovery is given in North (1965) [30].
} 
In a very real sense the job of cosmology is to provide a canvas upon which other fields of science, including particle physics, can weave their individual threads into the tapestry of our understanding of the Universe. Nowhere is the inherent unity of science better illustrated than in the interplay between cosmology, the study of the largest things in the Universe, and particle physics, the study of the smallest things ([24] p.362).

It is clearly implied here that particle physics is relevant for cosmology but the notion of interplay may also suggest a stronger notion of unity between the two disciplines. Kolb points out that '[t]he aim of modern cosmology is to understand the origin and the large-scale structure of the Universe on the basis of physical law' and there are those who take the ultimate foundation of physical law to be grounded in particle physics. Thus, it might be that particle physics could somehow be used to derive cosmology and thereby function as the 'material' of which the cosmological 'canvas' is made. ${ }^{2}$

Whatever the exact relation between cosmology and particle physics, Kolb seems to be talking about unity of science in a metaphysical sense of one common coherent picture of the world. But while this conception of unity may be methodologically useful, only a close inspection of the cosmology-particle physics interplay can determine to what extent such a grand metaphysical picture is in fact warranted. This state of affairs suggests a central question for the philosophy of cosmology which will be addressed in this article: What kind of unity obtains between particle physics and cosmology - for instance is particle physics (merely) relevant to cosmology or can particle physics be used to somehow derive cosmology? If an answer to this question can be provided, it should be interesting also in connection with more general unity claims in science - not least, of course, if Kolb is right that the interaction between cosmology and particle physics provides the best illustration of the unity of science.

In the following, I first turn to some general remarks on unity and reductionism. Second, in order to illuminate the nature of the cosmology-particle physics interplay, I review the observational support for the Big Bang theory, and discuss some of the conceptual issues which arise when particle physics is applied to cosmology - in particular the so-called cosmological constant problem and the conjecture of inflation. Short discussions are also included on other issues about the cosmologyparticle physics borderline such as the baryon asymmetry, dark matter, and the problem of understanding the very notion of the early universe. The final section summarizes and concludes.

\section{Unity With or Without Reductionism?}

The unity idea is often made explicit by referring to reductionism. At the ontological level reductionism amounts to the claim that entities and phenomena at a higher level can be reduced to those of a lower level, for instance in a chain like classical physics, atomic physics, particle physics, (string theory?). Within physics,

\footnotetext{
${ }^{2}$ This would be in line with Einstein's declaration from 1918: 'The supreme task of the physicist is to arrive at those universal laws from which the cosmos can be built up by pure deduction' 12].
} 
the discussion of reductionism is usually focused on the notion of emergence - the (ontological) question of whether new and non-reducible phenomena exist at the higher levels of description. For the discussion to follow, however, it will prove more useful to briefly review a more semantic or epistemological anti-reductionistic argument which can be associated with Bohr's insistence on the necessity of classical physics in the description of quantum phenomena.

Recall that, for Bohr, there is no doubt that material objects (e.g. a measuring apparatus) are composed of atoms. However, any description of an atomic system which involves a determination of its kinematical or dynamical variables must take the interaction of the system with a suitable measuring apparatus into consideration. Since a measuring apparatus, in order to yield unambiguous experimental results, is necessarily described by classical physics - e.g. no superpositions of pointer readings - the atomic (quantum) features of this apparatus must be ignored in a description of a quantum experiment. f There is little doubt that Bohr's views on the necessity of classical physics limits the intelligibility of ontological reductionism: if quantum physics does not make sense without classical physics, it is altogether unclear what one should understand by reducing classical physics to quantum physics.f

Bohr's view on quantum mechanics implies that the classical distinction between physical description and physical reality, e.g. in the sense of the description mirroring reality, will no longer do at the quantum level. The 'no mirror' situation is just another way of saying that the possibility of a full ontological reduction of classical objects to quantum objects is excluded: While atomic and sub-atomic objects exist independently of measurements, the (space-time/energy-momentum) properties of these entities do not, see e.g. [2]. If Bohr is right, it thus becomes impossible to see classical phenomena as merely a consequence of a deeper lying 'quantum reality'. Rather classical physics and quantum physics are both necessary for an account of our knowledge of nature (see also Falkenburg (1998) [13]). This argument against ontological reductionism does not exclude ontological unity - if the latter is merely taken to mean that entities and phenomena of two theories are interconnected (but not necessarily reducible to each other). Indeed, Bohr emphasized both that, on the

\footnotetext{
${ }^{3}$ See e.g. Hacking (1996) [15 and Hüttemann (1998) 19 for classification schemes of various kinds of unity, and Cat (1998) [9] for a detailed discussion of the relationship between unity, emergence, and (different notions of) reductionism in modern physics.

${ }^{4}$ Bohr argued that if the quantum features of the measuring apparatus, e.g. the uncertainties in its momentum and position, are taken into account, the 'quantum' results of the measurements, e.g. an interference pattern on a screen in the two slit experiment, are destroyed, see e.g. [2].

${ }^{5}$ Note that this is not in conflict with the formalistic fact that quantum expressions may correspond to classical expressions in certain limiting cases (e.g. when $\hbar \rightarrow 0$ ). In fact, as quantum theories are often constructed by 'quantizing' classical theories, it is not surprising that classical expressions may be formally derived from quantum expressions in some appropriate limit. Furthermore, Bohr's epistemological point would be unaffected even if one could establish more concrete links between the quantum and classical domain, e.g. through the notion of decoherence: if decoherence is taken to imply an explanation of certain classical features from a genuine quantum level, we would still be faced with the problem of making sense of this level (see also below). And, as Faye has noted, it is still far from clear what we should understand by a 'decoherence ontology' where '...a physical system in its indeterministic plurality, with its probability distributions and superposition, is the most fundamental entity in the world' (translated from the Danish) [14]. In any case, decoherence - as emphasized also by some of its spokesmen - does not by itself single out a particular interpretation of quantum mechanics, see e.g. Zurek [43].
} 
one hand, classical physics is necessary to define quantum phenomena, and, one the other hand, quantal laws are needed to explain the stability of classical objects.

As we shall see below, Bohr's arguments on the relationship between the classical and quantum level are connected to the discussion of the particle physics/cosmology interplay - in particular as concerns the question of whether cosmology may eventually be derived from a deeper (quantum gravity) theory.

\section{The Cosmology-Particle Physics Interplay}

The interplay between particle physics ideas and cosmology originated in work during the 1970s where it was realized that phase transitions associated with particle physics might have cosmological consequences (see e.g. the discussion in [35]). Nevertheless, the exchange between cosmology and microphysics in general is not just a product of modern particle physics. Indeed Gamov, in some sense the creator of the prevailing Big Bang paradigm, was led to his theory of the universe almost exclusively through nuclear physics, in connection with an attempt to account for the production of chemical elements - which seemed to require a very hot initial state (25 p.81,391). In the following subsections we will discuss to what extent this relevance of microphysics to cosmology, and vice versa, is observationally and/or experimentally supported when we move from nuclear physics to particle physics. While this move may appear as a straightforward change of scales (e.g. a move from considering neutrons and protons to considering the constituents of neutrons and protons) it involves the highly non-trivial change of theoretical framework from quantum mechanics to quantum field theory. A distinctive feature of quantum field theory is the peculiar concept of vacuum energy which, as we shall see below, plays a key role in the cosmology-particle physics interplay.

Intuitively, there seems to be a natural connection between cosmology and particle physics: Experiments in particle physics typically involve the study of reaction products from colliding high energy particle beams. The higher the energies, the heavier (and more exotic) the particles that can be produced in such collisions. Since the Big Bang is believed to have involved extremely high temperatures (energies), with the universe gradually cooling down from a hot and dense initial state, the early universe is portrayed as the ultimate particle physics laboratory. In fact, since recent speculations of unification in particle physics, e.g. that of grand unified theories and string theories, refer to energy scales which are far outside the range of particle accelerators on earth, the first tiny split second of the history of the universe apparently becomes the only domain in which such theories may be relevant. Although it is not easy to obtain information of the very early universe - for instance, the Big Bang universe was optically opaque at times early than 300,000 years (see below), setting a limit to how far back in time telescopes can probe - it is hoped that some relics from this early epoch can nevertheless be found in the cosmological data. Thus, cosmological observations might be used to constrain, if not test, speculative particle physics models. In turn, by addressing the nature of matter and forces at

\footnotetext{
${ }^{6}$ Although contemporary nuclear physics often involves quantum field theory, the nuclear physics calculations by Gamov and others, which were used to predict the light element abundances, were not made in a quantum field theoretic framework; see also below.
} 
extreme energy scales, such particle physics models may supply explanations for theoretical and observational puzzles in cosmology.]

If cosmology and particle physics are connected as outlined here, there must be some theoretical bridges between descriptions of the very small and the very large, or perhaps even a common framework for both disciplines. In the remainder of this section we shall focus primarily on two (closely related) theoretical bridges between cosmology and particle physics - the cosmological constant and inflation - which both turn out to throw doubt on the cosmology-particle physics connection. As a further probe of the relevance of particle physics to cosmology, we also briefly review the status of two examples where particle physics models are actively used in the search for answers to cosmological puzzles. Finally, we briefly comment on the conceptual problems in an attempt to provide a common framework for both cosmology and particle physics. But since the relation between cosmology and particle physics appears to rest on the assertion that the Big Bang model is effectively correct, due to the extreme energy conditions in the early universe, we first review the standard textbook evidence for the Big Bang in order to see where particle physics fits into the cosmological picture.

\subsection{Evidence for the Big Bang}

A central assumption in modern standard cosmology is that the Friedmann equation, derived from general relativity and describing the evolution of an expanding homogeneous and isotropic universe, has been at least approximately valid almost throughout the history of the universe. The faith in the Friedmann equation as the correct description of the universe dates back to Hubble's discovery in 1929 that other galaxies seem to move away from us, indicating that the universe is indeed expanding. This conclusion is often reversed and extrapolated as far as logic permits with the result that the universe must have been expanding from an initial singularity. But, strictly speaking, as the light from the most distant galaxies is supposed to have been emitted at a time when the universe was a few billion years old, the observed Hubble expansion does not say much about the very early universe. I

The time scale in the early universe is important, since the main observational evidence for the Big Bang, apart from Hubble's discovery, is the cosmic microwave background radiation, and the abundances of the light elements in the universe, each

\footnotetext{
${ }^{7}$ As the principal setting for the conjectured unity between cosmology and particle physics is the very early universe, we shall in this paper disregard questions concerning the role of particle physics in later astrophysical phenomena, for instance possible particle physics explanations of the so-called solar neutrino problem.

${ }^{8}$ Actually, what Hubble found was a law relating the distances to the galaxies to their observed redshift which was subsequently interpreted, through the familiar Doppler effect, as a universal expansion. We shall not here take up the interesting issue of justifying this interpretation which has been contested e.g. by adherents of the steady-state model.

${ }^{9}$ As we shall see later, however, even this logical extrapolation breaks down at least when a time around $10^{-43}$ seconds after the Big Bang is reached since classical general relativity is supposed to break down at so-called Planck scale energies where quantum gravity effects become important. This Planck time is the result of a purely dimensional consideration: it is the only time scale one can get by combining Planck's constant, the velocity of light and Newton's gravitational constant $\left(t_{\text {planck }}=\sqrt{G \hbar / c^{5}}=10^{-43} s\right)$.
} 
of which involves events at a specific point in time after the Big Bang. The Cosmic Microwave Background (CMB) radiation is believed to be a consequence of the decoupling between matter and radiation which occurred around $10^{13}$ seconds after the Big Bang (approximately 300,000 years). The hypothesized primordial nucleosynthesis, on the basis of which one can correctly predict the observed abundances of e.g. hydrogen and helium, is estimated to have taken place when the universe was roughly 1 second old (23] p.109). Schematically the evidence supporting the Big Bang, including the approximate time scale which the evidence probes, can be represented as follows:凹

\begin{tabular}{|c|l|l|}
\hline 1929 & Hubble expansion & $\sim 1$ billion years after Big Bang \\
\hline 1948 & $\begin{array}{l}\text { Gamov et al's prediction of } \\
\text { light element abundances } \\
\text { from nucleosynthesis }\end{array}$ & $\sim 1$ second after Big Bang \\
\hline 1965 & CMB radiation & $\sim 300,000$ years after Big Bang \\
\hline
\end{tabular}

But to reach an interaction point between particle physics and cosmology one has to extrapolate the Friedmann equation much further back than this evidence. Thus, the latest time where physicists expect a pure particle physics (quantum field theoretic) effect to have changed the cosmological situation is a mere $10^{-5}$ seconds after the Big Bang; namely where a phase transition associated with the theory of strong interactions is conjectured to have caused the recombination of hadrons, e.g. protons, from a primordial plasma of quarks and gluons. A phase transition (spontaneous symmetry breaking) associated with the electroweak theory is believed to have taken place still further back in time at around $10^{-11}$ seconds after the Big Bang. [12 Earlier times than $10^{-11}$ seconds (corresponding to higher energies) are well within the domain of speculative particle physics; but it is clear that even the domain of 'standard' particle physics (from, say, $200 \mathrm{MeV}$ to $300 \mathrm{GeV}$ corresponding to the range from $10^{-5}$ to $10^{-11}$ seconds after the Big Bang) is not what is probed by light element abundances - and less by the CMB radiation, and still less by light emitted from receding galaxies.[3]

\footnotetext{
${ }^{10}$ From the Friedmann equations for a radiation dominated universe (in thermal equilibrium) it follows that temperature in the early universe is related to time through $T \sim 1 / \sqrt{t}$. Note that this relation between time and temperature is not valid in the very early universe for non-radiation dominated epochs, e.g. during inflation.

${ }^{11}$ The specification of exact years should be taken with a grain of salt. As discussed in e.g. 25] Hubble did not in 1929 interpret his results as is done nowadays, nucleosynthesis was developed further after Gamov, Alpher and Herman's work, and the CMB discovery by Penzias and Wilson had a rich prehistory.

${ }^{12}$ It should be added that there is, so far, no direct experimental evidence in the laboratory for either the quark-gluon plasma or the electroweak phase transition (the latter is associated with the so-called Higgs field which, in spite of having being haunted for more than two decades, continues to escape detection).

${ }^{13}$ In fact, quantum field theoretic corrections to e.g. the conversion rate between neutrons and protons have been implemented in Big Bang nucleosynthesis models which predict the abundance of primordial Helium-4, see e.g. [28]. If one therefore had a sufficiently accurate observational determination of this primordial abundance, one might establish a more direct connection between quantum field theoretical effects and cosmological observations about the time of primordial nucleosynthesis. However, it appears that recent observational determinations of the primordial Helium-4 abundance are both controversial (different methods yield inconsistent results) and too
} 
Although the observational pillars supporting the Big Bang thus provide no direct support for the connection between cosmology and particle physics, they may be used to constrain speculative particle physics models. Examples of such 'constraining' cosmological data include: (i) the light element abundances, which through their agreement with nucleosynthesis constrain the number of neutrino species; and (ii) anisotropies in the CMB which can be interpreted as relics from earlier epochs and therefore constrain parameters e.g. in various inflation models (see e.g. 40]). But while cosmological data may provide constraints on model building in particle physics, these constraints can hardly constitute a test of the relationship between the disciplines. For instance, the nucleosynthesis constraint on the number of neutrino species arises since more neutrino species would increase the primordial production rate of Helium-4. Thus, some speculative particle physics model which predicted more neutrino species than allowed from the nucleosynthesis constraint would be in trouble from the outset. However, as there is no theoretical prediction (or explanation) of the number of neutrinos in standard particle physics (the 'Standard Model'), it seems reasonable to maintain that the constraint from nucleosynthesis cannot be seen as a cosmological test of a particle physics prediction. Of course, since nobody would deny that nuclear physics is related to particle physics, it would be foolish to assume that the Big Bang nucleosynthesis model is correct but that no particle physics is involved (in the finer details of the model). The argument made here, however, is only that there is so far no direct evidence, for instance from the confrontation between nucleosynthesis predictions and observed abundances of the light elements, which qualifies as a cosmological test of the particle physics before (or at) the time of nucleosynthesis.

From this short discussion it seems in any case clear that the kind of unity which particle physics and cosmology may possess must be quite different from that of e.g. electricity and magnetism - which holds for our present epoch without any recourse to a historical development, and which has direct and manifold experimental and observational consequences. We now turn from Big Bang evidence to some of the more conceptual problems to be overcome in establishing a connection between cosmology and particle physics.

\subsection{The Problem of the Cosmological Constant}

Arguably, the most important theoretical prerequisite for unity between cosmology and particle physics will be a suitable consistency between general relativity (GR) and quantum field theory (QFT). But here, there arises an important problem known as the problem of the cosmological constant. In essence the problem is that whereas the cosmological constant in GR is experimentally known to be zero or close to zero,

imprecise to distinguish quantum field theoretic corrections in the nucleosynthesis models; see e.g. 37.

${ }^{14}$ The first part of the present subsection is based on a manuscript, co-authored with Svend E. Rugh, which provides a detailed analysis of the historical and conceptual aspects of this problem, as well as the physics on which it relies [35. In the following, most details of this analysis are omitted in an attempt to give an overview of how the cosmological constant problem is related to the question of unity between cosmology and particle physics. 
QFT predicts an astronomically high value for this quantity. 'cosmological' constant in particle physics is due to the fact that the vacuum energy in QFT takes the form of the cosmological constant in Einstein's equations.

While the vacuum energy can be explained away in QFT since only differences in energy matter, GR is sensitive to any absolute value of this vacuum energy. Thus a huge vacuum energy in GR would wrap up all of spacetime at macroscopic scales - in striking contrast to what is in fact observed. More technically, Einstein's equations read

$$
R_{\mu \nu}-\frac{1}{2} g_{\mu \nu} R-\Lambda g_{\mu \nu}=-\frac{8 \pi G}{c^{4}} T_{\mu \nu}
$$

where $R_{\mu \nu}$ and $R$ refer to the curvature of spacetime, $g_{\mu \nu}$ is the metric, $T_{\mu \nu}$ the energy-momentum tensor, $G$ the gravitational constant, and $c$ the speed of light. A non-zero value of Einstein's cosmological constant would lead to a curvature of 'empty' space, i.e. when no matter or radiation is present $\left(T_{\mu \nu}=0\right)$, and it is therefore usually assumed that $\Lambda$ receives contributions from any energy which might be present in vacuum. ${ }^{\text {m }}$ In particular, the quantum expectation value of the vacuum energy density $\left(\left\langle\rho_{V a c}\right\rangle\right)$ in particle physics, which is composed of the vacuum energy of all the fields in QFT, is supposed to act as such a contribution to the cosmological constant:

$$
\Lambda=\Lambda_{0}+\frac{8 \pi G}{c^{4}}<\rho_{v a c}>
$$

where $\Lambda_{0}$ can be referred to as a 'bare' cosmological constant (representing any 'non-standard' vacuum energy which would curve empty space when no matter or radiation is present). Each of the individual terms which build up the vacuum energy density in QFT is estimated to be incredibly much larger, approximately $40-$ 120 orders of magnitude, than the value of the cosmological constant allowed from observation. The discrepancy, in turn, can only be circumvented by assuming that some mysterious cancellations take place in the total contribution to the cosmological constant (either between the individual contributions to QFT vacuum energy or between the terms in eq.(2) ) - cancellations which, on our present understanding of QFT and GR, seem highly implausible.

Note that the introduction of quantum vacuum energy, which rests on the notion of a fixed (Minkowski) spacetime background, in a classical equation, which associate matter and energy with a dynamical (non-Minkowski) spacetime, represents a conceptual clash between two altogether different frameworks. It is therefore not self-evident whether such a semi-classical approach, which involves the difficult issue of treating quantum fields in curved spacetime backgrounds, is reasonable for even formulating a cosmological constant problem. In fact, as discussed in detail in 35],

\footnotetext{
${ }^{15}$ Recent observations on supernovae have indicated that the cosmological constant in GR is small but non-zero. In fact, one may distinguish three different "cosmological constant problems": The observational question of the value of the cosmological constant, the problem of the GR-QFT clash dealt with here, and an 'expected-scale' problem saying that if the possible successor to the Standard Model is a quantum theory of gravity characterized by the Planck mass $m_{p}$, one would naively expect the observed cosmological constant to be of order $m_{p}^{4}$.

${ }^{16}$ Einstein originally introduced his 'anti-gravity' constant to secure a static universe. Not least after Hubble's observations, which indicated an expanding universe, Einstein became very sceptical about the constant and is reported to have called it 'the biggest blunder of my life'; see also [32].
} 
the notion of a cosmological constant problem rests fundamentally on two assumptions - both of which can be questioned: (i) The QFT vacuum energy is physically real (as in the standard QFT interpretation); and (ii) the semi-classical approach, in which quantum energy acts as a cosmological constant, is valid. As is evident from eq.(2), this last assumption implies a theoretical bridge between GR and QFT. It can be argued that curved spacetime effects on QFT are mostly small - this is the case e.g. in our local astrophysical neighbourhood where the gravitational field is rather weak - and hence that the vacuum energy is reasonably well defined in the semi-classical context (this provides some justification for (ii)). So the estimate of vacuum energy in standard QFT appears in any case to be a severe obstacle to compatibility between GR and QFT. Consequently, the cosmological constant problem threatens the inter-theoretical consistency between cosmology and particle physics.

However, it can also be argued that the cosmological constant problem, instead of being a problem for unity, points in the direction of further unification in terms of some quantum theory of gravity. In fact, the most promising solution to the cosmological constant problem has sometimes been believed to lie in the framework of quantum cosmology, in which quantum theory is applied to the universe as a whole. ${ }^{18}$ Quantum cosmology is usually based on the so-called Wheeler-DeWitt equation for the 'wave-function of the universe', which results from the canonical approach to quantum gravity where general relativity (the metric tensor) is quantized, see e.g. (《8 p.65). [9 The idea of a quantum cosmological solution to the cosmological constant problem, which was first suggested by Hawking in 1984 and further elaborated by Coleman in 1988, is to show - by introducing a new 'quantum cosmological' gauge field or using bizarre features of 'quantum' spacetime known as wormholes - that the value of the cosmological constant with very high probability must be zero in our universe (see e.g. 41 p.20 ff. for discussion and references). We shall not enter into any details of this proposal and its specific problems but rather, in connection with the question of unity, point out some of the general difficulties in using a quantum cosmological framework for whatever purpose.

Although no consistent quantum theory of gravity, and therefore no consistent quantum cosmology, exist as yet, one can give some more or less heuristic arguments in favour of why such theories are eventually to be expected. For instance, it may appear strange that gravity, as a force acting on matter which can otherwise be described quantum mechanically, should not itself be quantum in nature. A stronger

\footnotetext{
${ }^{17}$ As the name suggests, quantum gravity treats gravity in a quantum manner, but there exist a number of different approaches for how to do that, see e.g. Butterfield and Isham (1999) (『8] p.39 ff.). The 'degree' of unification attempted in the various approaches to quantum gravity varies - some approaches, like string theory, necessarily imply unification of the gravitational force with the strong and electroweak forces, whereas other approaches, e.g. the loop quantum gravity programme, do not (《8], p.66).

${ }^{18}$ Although there is so far no generally accepted solution to the cosmological constant problem, a number of different ideas has been tried (see [35] for a discussion and classification of the suggested solution types). We shall here restrict attention to the quantum cosmology proposal as this theory arguably provides the most radical attempt of joining cosmology and quantum mechanics but it should be noted that the most famous example of a quantum gravity, string theory, is also plagued by the cosmological constant problem - so far with no cure in sight.

${ }^{19}$ In contrast to quantum cosmology, quantum gravity - which may be characterized as any attempt to join general relativity with quantum mechanics (or quantum field theory) - might also have applications other than for early universe cosmology, e.g. black hole physics.
} 
argument along similar lines has been given by a leading quantum cosmologist, Hartle, who phrases his trust in a quantum theory of gravity in an almost logical, if slightly paradoxical, form:

It is an inescapable inference from the physics of the last sixty years that we live in a quantum mechanical universe - a world in which the basic laws of physics conform to that framework for prediction we call quantum mechanics. If this inference is correct, then there must be a description of the universe as a whole and everything in it in quantum mechanical terms. (16] p.67)

In the second sentence, Hartle makes two claims: 1) that there must be a quantum description of the universe as a whole, and 2) that it must be possible to describe everything in the universe in quantum mechanical terms. The first of these claims is a claim of the necessity of quantum cosmology; the second claims the necessity of quantum gravity - insofar as Hartle takes it for granted that everything, besides gravity, has already been described by quantum mechanics. Although, as indicated above, these enterprises are closely related, we shall here just comment on quantum cosmology, and return later to some remarks on the possibility and necessity of quantum gravity.

First, in spite of Hartle's historical 'evidence', it is questionable whether there is an 'inescapable inference' from the spectacular successes that quantum mechanics has had in the microscopic domain to a statement that the whole universe is genuinely quantum mechanical - even taking into account that quantum theory can be applied to some macroscopic systems such as superconductors.20 For one thing, insofar as the concept of a 'wave-function of the universe' is at all a sensible concept, its governing Wheeler-DeWitt equation is "horribly ill-defined in any exact mathematical sense" (see e.g. [8] p.65).

Another peculiarity of quantum cosmology, related to the (lack of?) meaning of the wave function, is the absence of a precise notion of measurement due to the lack of a clear distinction between the observer and the system to be observed: contrary to standard quantum mechanical systems there is obviously no 'external' system to the universe! In the discussion that follows Hartle's quote above, he indicates that quantum cosmology therefore poses a problem for the 'Copenhagen' interpretation of quantum mechanics (《16] p.67).27 Nevertheless, one could also argue the other way around: If Bohr's insistence of the necessity of a classical framework for understanding quantum mechanics is correct, then quantum cosmologists, not Bohr, have a problem. A resolution of this issue depends on which, if any, of the following is true: 1) the interpretation of quantum mechanics is a matter of choice to fit the needs of the situation; or 2) one can give general arguments against certain interpretations; or 3) there are arguments of principle to single out one interpretation.2 Although

\footnotetext{
${ }^{20}$ That this inference may not be straightforward seems to be implicit also in Halliwell's nontechnical introduction to quantum cosmology [17, see especially p.488. For more reservations on the universal validity of quantum mechanics, see e.g. Hendry (1998) [18].

${ }^{21}$ Quantum cosmologists often invoke Everettian views on quantum mechanics backed by the notion of decoherence, see e.g. 21] p.10 and [7] p.38.

${ }^{22}$ See Faye 14 for a discussion of problems with so-called realistic interpretations of quantum mechanics, and P. Zinkernagel 42 for an account of how the necessity of a classical framework can be understood in a broader philosophical framework of 'conditions for description'.
} 
this is not the place to go into this much debated subject, it seems clear that Bohr's arguments, briefly discussed in the previous section, demonstrate that the notion of a 'quantum reality' implied by the wave-function of the universe might very well be unintelligible (see also below). In any case, it is clear that discussions of quantum cosmology, for instance in connection with the cosmological constant problem, take us right back into the philosophical battlefield of interpreting quantum mechanics.

Finally, even if we by-pass these conceptual issues for a moment, the chances of actually observing quantum cosmological effects, characterized by the Planck scale, seem remote. This situation is illustrated by the fact that an extrapolation from the supposed time elapsed from the Big Bang until nucleosynthesis (1 sec) back to the Planck time $\left(10^{-43} \mathrm{sec}\right)$ is considerably longer on a logarithmic scale than an extrapolation from the present $\left(\sim 10^{17} \mathrm{sec}\right)$ back to the time of nucleosynthesis! In this connection, it should be noted that chances for testing aspects of quantum cosmology may be further weakened by, ironically, another aspect of early universe speculation, namely inflation. This is because an inflationary period may well wipe out all traces of a pre-inflationary (quantum cosmological?) epoch, see e.g. ([23] p.458).

\subsection{Inflation}

Inflation is short-hand for the idea that the expansion of the universe at an early stage in its history proceeded extremely fast. In turn, such a period of rapid expansion may provide a solution to some conceptual problems in standard Big Bang cosmology (see below). Originally, the idea of inflation was conceived of as a consequence of the vacuum energy associated with a spontaneous symmetry breaking in the Grand Unified Theory (GUT), according to which the strong force and the electroweak force will be unified at energies above $10^{14} \mathrm{Gev}$ (in the simplest version of the theory). This energy corresponds to a time $\sim 10^{-34}$ sec after the Big Bang and thus inflation was a clear candidate for a solid contact point between cosmology and particle physics.23 One way in which inflation has been imagined is that during a short 'inflation' period, lasting some $10^{-32}$ seconds, the huge vacuum energy associated with a GUT symmetry breaking field effectively acts as a cosmological constant. This anti-gravitational vacuum energy boosts the expansion of the universe exponentially until the vacuum energy is finally converted into heat and the universe enters the standard (slowly expanding) epoch.

Since vacuum energy is the source of almost all inflationary scenarios, there is an explicit connection between the cosmological constant (problem) and inflation: the vacuum energy of some specific field was "good" at early times - inflation runs on it so to speak - but vacuum energy in general is not so good now, for we know it must be very small or zero. This connection is further illuminated since inflation, just like we noted above for the cosmological constant problem, is cast in a semi-classical framework: Due to its (anti-)gravitational effect, quantum vacuum energy is driving a classical general relativistic expansion of the universe. In this way inflation makes

\footnotetext{
${ }^{23}$ The conversion between time and energy is found from the Friedmann equation using the curious equation of state for a vacuum energy dominated universe, (energy density) $=-$ (pressure), see e.g. (23] p.270 ff). The negative pressure associated with positive vacuum energy density also explains how that latter gives rise to a repulsive, anti-gravity, effect, see e.g. [35].
} 
the cosmological constant problem even more disturbing, and the latter has therefore raised some critical voices on the inflation speculations:

It seems difficult to understand the cosmological constant as a vacuum energy density now, and it is therefore naive to exploit it this way during the first $10^{-35}$ seconds of the universe (Boerner 1988) [1].

Also cosmologists with a more optimistic attitude to the idea of inflation acknowledge that the connection between the cosmological constant problem and inflation may turn out to be fatal. Thus Kolb and Turner writes:

...that puzzle [the cosmological constant problem] is a potential Achilles heel for the scenario: If some grand principle should be discovered that renders vacuum energy at all times impotent, inflation would lose the ultimate source of its power (23 p. 314)

Even if vacuum energy based inflation might seem to be in trouble as a reasonable theory from the outset, the idea is claimed to have provided cosmology with possible solutions to the horizon, the flatness, and the monopole problems.20 However, as a theory covering only the first split second of the history of the universe, inflation's epistemic status is problematic (even if not as problematic as that of quantum cosmology). For instance, Narlikar noted in 1987 that 'The absence of monopoles, domain walls, seeds of galaxies, and the rest is confidently taken to support a number of hypotheses regarding the unification theory and the very early universe. Nowhere else in physics have null observations been used to claim so much that cannot be otherwise tested' [29]. Now, Narlikar is one of the few who still defends a competing world view - that of a 'quasi' steady state model, a world without a beginning in a Big Bang - and so one might be hesitant about accepting his challenge to inflation [6]. Regardless of world view preferences, however, Narlikar is surely right that it is not particularly impressive if the observational 'evidence' of a theory is taken to consist of what is not seen.

But there might of course be more than 'null observations' which could support inflation. To this end, it is often emphasized that a generic prediction of inflation is an almost scale invariant spectrum of the anisotropies in the microwave background radiation. ${ }^{2}$ Since this is actually what is seen in the data, the anisotropy spectrum is a potential sign of inflation, although other cosmic scenarios may lead to similar effects (4 p.19). This emphasis on anisotropies in the microwave background radiation, however, can be a double-edged sword: For instance, all GUT based inflation models generically predict the amplitudes of these anisotropies to be far higher than microwave background data allow - and, in general, '... arranging for acceptable density perturbations [directly linked to the anisotropies] is the most restrictive

\footnotetext{
${ }^{24}$ Attempts have been made to solve the cosmological constant problem in the context of quantum

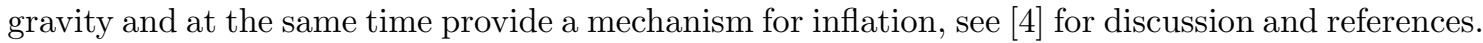
Given the provisional nature of quantum gravity, however, these attempts are, at best, incomplete.

${ }^{25}$ These problems concern, respectively, the question of why the background radiation is isotropic, why the observed universe is Euclidean to a very high degree, and why no monopoles are observed (these monopoles are objects which are expected on GUT grounds). See Earman and Mosterín (1999) for a critical analysis of inflation and its merits as regards solving these problems [11].

${ }^{26}$ For more discussion of generic predictions of inflation, e.g. that of spatial flatness, see [11].
} 
constraint on inflationary potentials (and thereby the underlying particle physics models)' (23 p.288 and 295). The observational status of inflation is furthermore complicated by the fact that the inflation industry has produced a vast number of models which easily accommodate a wide variety of observational situations:

The trouble is that inflation is a paradigm rather than a model [...] a reasonable first guess is that at present there are around one hundred different models on the market, all consistent (at least more or less) with present observational data. (Liddle 1999 [26])

Although it is therefore difficult to either support or rule out the inflation idea by means of observations, planned detailed studies of the microwave background in the near future are expected to cast more light on the situation. ${ }^{27}$

Worse than the observational issue, perhaps, for the relation between cosmology and particle physics, are the developments in inflation models which have shown that such models are in no obvious way related to the spontaneous symmetry breaking of a particle physics model. Already in 1983, Linde suggested a so-called 'chaotic' version of inflation which operates with a very simple potential to drive the inflationary period (see e.g. 27 p.66). While the simplicity of the inflation field (the 'inflaton') in Linde's model does not exclude some particle physics origin of it being found, Linde made no attempt to link his inflaton to particle physics. The chaotic inflation scenario thus demonstrated, in contrast to the spirit of Guth's original 1981 proposal, that inflation by no means has to have a particle physics origin. In accordance with his view on unity, however, Kolb complains that although models such as chaotic inflation realize the 'true essence' of inflation without complicating details of underlying physics, chaotic inflation "...is not "realistic" in the sense that no one would accept the existence of a scalar field whose sole purpose is to make inflation simple...' (24 p.32). And, indeed, while Linde's proposal is still an area of active research, there is also an ongoing tradition among imaginative physicists in search of a basis for inflation in speculative particle physics models (see e.g. [4]).

\subsection{Other Problems on the Borderline?}

In this subsection I briefly mention a few other topics on the borderline between cosmology and particle physics. Although the issues mentioned below do stimulate activity in both fields, they do not unambiguously speak in favour of their being intimately related. The following list is not intended to be exhaustive but it illustrates that there are more puzzles on the way to unity than 'just' the cosmological constant and inflation.

Baryon-asymmetry. Observations of the universe indicate that the amount of antimatter (anti-baryons) is negligible in comparison to matter (baryons), so that the universe seems to be baryon-antibaryon asymmetric (see e.g. 23 p.158). Moreover, it turns out that the correct predictions of the light element abundances in the

\footnotetext{
${ }^{27}$ Recent results from the balloon experiments Boomerang and Maxima seem to be consistent with a spatially flat universe (which is predicted by most inflation models) [20].
} 
universe from the theory of nucleosynthesis depend directly on the parameter $\eta$ which characterizes the baryon asymmetry.28

On the other hand, if a particle physics model is to generate such a surplus of baryons over anti-baryons; the obvious, though not the only, condition is that the usual rule of baryon charge conservation must be violated.29 And although processes where baryon charge is not conserved have never been seen experimentally, physicists began to consider them seriously with the advent of grand unified theories in the 1970s in which such processes appear naturally [10]. However, simple versions of GUTs are now ruled out precisely because baryon charge non-conservation processes, in particular the decay of the proton, have still not been seen in spite of intensive searches (the simple GUTs predict a lifetime of the proton which is incompatible with lower limits from experiments). Nevertheless, for some cosmologists, the observational fact of a baryon asymmetry is still taken to support the cosmology/particle physics relationship:

The baryogenesis scenario is one of the great triumphs of particle cosmology, and in the absence of direct evidence for proton decay, baryogenesis may provide the strongest, albeit indirect, evidence for some kind of unification of the quarks and leptons [as in GUTs]. (Kolb 1993 [23] p.158)

But apart from the fact that baryogenesis has been proposed in non-GUT scenarios, it is not clear that the asymmetry is in particular need of an explanation from a general relativity based cosmological point of view in the first place. Only if the quantum mechanical fact that each particle has an anti-particle is taken into account, and if it is assumed that quantum mechanics rules in the very early universe (whatever that means), does the necessity of an explanation arise. Indeed, to seek an explanation of the baryon asymmetry is connected with the assumption that particle physics is somehow the place to find cosmological answers, as noted in a recent review of electroweak baryogenesis:

We could just accept this [the baryon asymmetry] as an input parameter for the evolution of the universe. However it is part of the philosophy of modern cosmology to seek an explanation for the required value of $\eta$ using quantum field theories of elementary particles in the early universe. (Trodden 1998 [39])

\footnotetext{
${ }^{28} \eta$ is defined as the ratio between the excess of baryons over anti-baryons to photons and is supposed to have remained roughly constant since the time of primordial nucleosynthesis. Direct observations of baryonic matter in the universe only account for one third of the value of $\eta$ required by successful primordial nucleosynthesis, but the discrepancy is thought to be due to the presence of large quantities of dark or invisible baryonic matter, see (10] p.3) and also below.

${ }^{29}$ Sakharov (1967) [36] was the first to discuss the necessary features of a particle physics model to generate the baryon asymmetry.

${ }^{30}$ The idea of electroweak baryogenesis, which is currently the most popular scenario for generating the baryon asymmetry, is to explain the baryon charge non-conservation on electroweak energy scales, through so-called non-perturbative processes in the electroweak theory. However, this scenario for baryogenesis is only viable when non-tested extensions of the electroweak model, for instance supersymmetry (a symmetry between fermions and bosons), is included in the description 39.
} 
This is obviously a statement of choice but it is clear that once the cosmological observed baryon asymmetry is supposed to be explained by particle physics, the hitherto lack of generally accepted scenarios based on experimentally tested ideas makes it hard to judge whether the baryon asymmetry is good or bad news for the connection between cosmology and particle physics.

Dark matter. Like the baryon asymmetry, this issue has its origin in astronomical observations. The hypothesis of dark matter arises as a response to the problem of accounting for various astronomical phenomena which seem to require much more mass than is observed. A well-known example is the case of observed rotation curves for galaxies: the estimated mass of luminous matter in the galaxies cannot account for the observed rotation velocities of objects far from the galaxy center (see e.g. [23] p.16-19). As mentioned above, some of this dark matter must be baryonic, e.g. in the form of white dwarfs, neutron stars, diffuse warm gas or even black holes, to be consistent with the predictions about nucleosynthesis. From comparisons between the primordial nucleosynthesis scenario and observed abundances of e.g. deuterium, it follows that the total amount of baryonic matter in the universe is $\Omega_{\text {baryon }} \sim 0.03$, but since estimates on the total amount of matter in the universe (e.g. from studies of the density and properties of galaxy clusters [40]) suggest that $\Omega_{M} \sim 0.2-0.4$, one also needs non-baryonic dark matter components. 11 Nevertheless, precise estimates of the matter content in the universe are difficult to make, and there are still those who think that all matter (dark and luminous) may, in fact, be baryonic, see e.g. [3] and (223 p.369ff).

Particle physics has a number of candidate models for what this dark matter could be. Popular models include those of axions, supersymmetric particles or massive neutrinos - all of which are characterized by the fact that they go beyond standard particle physics and they refer to particles which are believed to have been produced in the early universe. From observational constraints it seems that massive neutrinos, denoted as 'hot' dark matter, can at most account for a small fraction of dark matter in the universe. This suggests that 'cold' dark matter, for instance axions or supersymmetric particles, is the dominant component of this invisible matter (see e.g. Turner and Tyson [40]).

While Turner and Tyson take observations to be a suggestive pointer to the existence of nonbaryonic dark matter, another leading cosmologist has this to say on the issue:

In short, I don't think we can argue that nonbaryonic cold dark matter has proved to be considerably more stable than the phlogistical chemistry that occupied much of Henry Cavendish's scientific career [...]. (Peebles 1999 [31]). 2 [2]

\footnotetext{
${ }^{31}$ We have here used the convenient way of expressing the amount of a certain type $x$ of mass or energy in the universe through the dimensionless parameter $\Omega_{x}$. This is defined by $\Omega_{x}=\rho_{x} / \rho_{c}$, where $\rho_{x}$ is the mass/energy density of $x$, and $\rho_{c}$ corresponds to the critical mass/energy density in the universe which distinguishes open, flat, and closed universes (i.e. if $\rho_{\text {total }}=\rho_{c}$ and thus $\Omega_{\text {total }}=1$, then the universe is flat).

${ }^{32}$ Peebles has remarked, however, that he is somewhat more optimistic regarding cold dark matter after the Boomerang and Maxima results which were mentioned above (private communication).
} 
In addition to the nonbaryonic dark matter question, the usual theoretical expectation from inflation is that $\Omega_{\text {total }}=1$, and thus the total estimated matter content of the universe is, even with new exotic forms of dark matter such as axions, insufficient in relation to this (almost generic) inflation prediction. So to make matter estimates compatible with inflation expectations, particle cosmologists contemplate that a tiny part of the vacuum energy which once drove the rapid expansion of the universe may still be with us.35

As with the baryon asymmetry, the question of the relationship between cosmology and particle physics remains unanswered: while cosmological observations can constrain the models suggested by particle physics, none of the particle physics candidates for dark matter have been clearly demonstrated in cosmological observations or in particle accelerators. matter - as well as the conjecture of small amounts of vacuum energy of unknown source- can therefore, according to taste, be regarded either as a problem for the connection between cosmology and particle physics or as an important motivation for further scrutinizing cosmological observations and speculative particle physics models.

Defining space and time. In modern cosmology the popular question "what was before the Big Bang?" is mostly put aside as meaningless with the argument that time and space were created with the Big Bang. But since the principal scene for a unification between cosmology and particle physics is the very early and very small universe, reasonable notions of time and space are obviously required right after the beginning for the unification to make sense.

It is usually assumed that the backwards extrapolation of the Friedmann equation becomes invalid at the Planck time, $t \sim 10^{-43}$ sec, where it is expected that quantum effects of gravity will make classical general relativity break down. As indicated earlier, theories of quantum gravity attempt to unify or reconcile general relativity with quantum field theory but it is still not clear how, or if, it is possible to achieve a theory encompassing both Einstein's theory, where matter is treated classically and spacetime is dynamical, and the quantum field theory of matter which is formulated in a fixed spacetime background.5 In spite of this, it is sometimes assumed that quantization of the gravitational field is, in fact, a necessity due to analogies with the famous 1933 Bohr-Rosenfeld analysis of the electromagnetic field quantization (see e.g. [21]). Rosenfeld has pointed out, however, both that the analogy between the gravitational and the electromagnetic field is problematic (due to the appearance of a definite scale for space and time intervals in the theory of gravity), and that the Bohr-Rosenfeld analysis showed only the consistency of the electromagnetic field quantization, not its necessity ([33], see also [38] p.235).

Although the treatments of space and time must be closely connected in a rela-

\footnotetext{
${ }^{33}$ As indicated above, recent observations of supernovae seem to require a small value of $\Lambda$ $\left(\Omega_{\Lambda} \sim 2 / 3\right)$, consistent with $\Omega_{\text {total }}=1$, see e.g. 40 .

${ }^{34}$ There is some evidence that one or more of the neutrinos may have a non-zero mass but, as mentioned above, this is of little help since hot dark matter is not believed to be the whole story.

${ }^{35}$ Recent developments in quantum gravity suggests that in order to perform this reconciliation, it might be necessary to abandon the conventional ideas of quantum field theory of depending on a metric structure [34] - but is by no means clear how this would affect the successes of standard QFT, which appear to be founded on the idea of fields defined on a (fixed) spacetime background.
} 
tivistic framework, we shall here just comment briefly of the possibilities of defining time in the "early" universe. Even if a theory of quantum gravity - which presumably governs the very early universe - could be found, it is notoriously difficult to see how the notion of time could somehow emerge from such a theory. For instance, the canonical Wheeler-DeWitt equation of quantum gravity does not depend on time which obviously makes it hard to see if it can at all describe an evolving universe (see e.g. [23] p.457). The problem of how time might emerge from quantum gravity is discussed in detail by, for example, Butterfield and Isham [7]. These authors point out (p.57) that the emergence of time in quantum gravity is not, in fact, a process in time, so that contradictory questions such as 'how did the universe look before time emerged' can be avoided.

Nevertheless, even if quantum gravity can avoid downright contradictions, there are at least two reasons to doubt whether such a theory could be relevant for the description of our (early) universe. First, although there might be no temporal relation implied by the emergence of time from quantum gravity, it is still highly uncertain how, and in what sense, time may be said to emerge from quantum gravity - not least because even semi-classical approximations of the theory, where a time parameter might be defined, are beset with technical and conceptual problems (see e.g. [7] pp.49-52 and references therein). 30 Second, apart from this well-known problem of how time can emerge in the theory, it is also hard to make sense of the "reverse" transition from time in the early universe to a timeless quantum gravity. This problem, which could be called the reverse problem of time, arises as follows: One of the fundamental ingredients in the Big Bang scenario is the existence of a cosmic time $t$ which is obviously necessary for discussing various epochs of the universe. The cosmic time parameter is the proper time of a standard (classical) clock at rest in the so-called comoving frame (see e.g. [24), and it is this time concept cosmologists have in mind when discussing conditions in the early universe. Thus, the assumption that quantum gravity (or quantum cosmology) is relevant for the study of the very early universe rests on a solid classical notion of time. But if it is conjectured that timeless quantum gravity is the fundamental theory, it appears paradoxical that its central field of application (the early universe) is only defined by a concept (classical cosmic time) which is completely alien to the theory! In turn, if we cannot even discuss the central application of quantum gravity without assuming a classical time concept, it is unclear what we should understand by an assumption like 'the ultimate nature of spacetime is non-classical' and, more generally, what we should understand by the assumption that 'classical physics can ultimately be reduced to quantum gravity'.

In connection with their discussion of quantum gravity and the 'Copenhagen' interpretation of quantum mechanics, Butterfield and Isham note ([7] p.37):

...we admit to giving this sort of interpretation little credence. For we see no good arguments for the necessity of such a background manifold

\footnotetext{
${ }^{36} \mathrm{An}$ aspect of these problems concerns the difficulties involved in a straightforward application of decoherence in the quantum cosmological context (as in e.g. 21] p.24-26). Specifically, it is difficult to see how decoherence, which in standard quantum theory is construed as a process in time, can be relevant to recovering the usual time concept when the underlying quantum gravity theory is timeless ([7] p.52).
} 
[classical spacetime] as, for example, (in Bohr's words) a 'precondition of unambiguous communication'.

But, as we have just seen, even if it is assumed that quantum gravity is relevant for cosmology, one can in Bohrian terms say that a classical (cosmic) time indeed seems to be a necessary condition for unambiguous discussions of the early universe.

These brief remarks can in no way do justice to the profound conceptual problems with which a quantum theory of gravity is faced, but - like the cosmological constant problem issue - they illustrate that the search for a unified quantum gravity theory is not necessarily a viable road to unity between cosmology and particle physics.

\section{Summary and Conclusion}

So far, there is little or no observational evidence which motivates the idea that particle physics is intimately related to cosmology. Although cosmological data can constrain speculative particle physics models which might explain features of the present universe, these models have yet to receive direct experimental or observational support - either in physics laboratories or in cosmological observations of clear footprints from a particle physics dominated era in the early universe. Thus, although the observed baryon asymmetry and the apparent need for dark matter have led to interesting theoretical ideas beyond standard particle physics, these ideas are still purely speculative. And even the cosmological consequences of relatively well established particle physics remain theoretical conjectures. In particular, the observational pillars of the Big Bang provide no direct support for the cosmologyparticle physics connection - simply because the scene for this connection is much earlier than the epoch of nucleosynthesis, the release of microwave background radiation, and the formation of galaxies which eventually have permitted cosmologists to observe the Hubble expansion. Thus, it has yet to be established that particle physics is relevant to cosmology.

On the more theoretical side, we first reviewed the cosmological constant problem which arises due to the assumed relation between (physically real) quantum vacuum energy and general relativity. While various types of solutions to this problem are conceivable, we discussed some of the difficulties associated with construing solutions in a unified framework of quantum gravity. In particular, we noted with respect to quantum cosmology that 1) as yet, there exists no consistent quantum theory of gravity and thus no quantum cosmology, 2) dealing primarily with the very early universe it would be difficult to test such a theory, and 3) it is still far from clear that it makes sense to apply quantum mechanics to everything in the universe or to the universe as a whole. Given these general problems about imagining a solution of the cosmological constant problem provided by a further unified theory, the problem remains a source of doubt as to whether general relativity and quantum field theory are at all compatible. Consequently, the cosmological constant problem is a problem for the consistency between cosmology and particle physics, at least insofar as the latter is taken to involve physically real vacuum energy.

As far as inflation is concerned, we have seen that this theory does not necessarily provide optimism for an intimate connection between cosmology and particle 
physics; for three reasons. 1) The physical mechanism for inflation, as a rearrangement of the vacuum structure in the early universe, is obscured by the cosmological constant problem which tells us that the concept of vacuum is still not well understood. 2) Inflation is epistemologically doubtful: Since inflation only covers the first split second of the universe it is difficult to test, and, conversely, the huge variety of inflation models which can accommodate a wide range of observational results makes it difficult to rule inflation out. 3) While inflation was originally based on simple versions of Grand Unified Theories and so was a "good move" in the unification scheme, we now know that inflation cannot be based on such theories (which are also most likely wrong), and that some later inflation models do not operate with quantum field-based inflation ideas. It is thus no longer clear that a period of cosmological inflation can be derived from fundamental physics.

As a last theoretical issue we have mentioned - although by no means discussed in detail - that the differences in the treatment of spacetime in quantum field theory and general relativity, as well as the problematic notion of time in prototype versions of quantum gravity, could mute optimism for finding a unified theory in which cosmology and particle physics would be treated in a common framework. This point makes it doubtful whether cosmology could eventually be derived from particle physics in some appropriate limit of quantum gravity.

With hesitations about both the relevance of particle physics to cosmology, the consistency between GR and QFT, and the possibility of deriving cosmology from a deeper quantum (gravity) theory - let us return to the question of what kind of unity can be claimed for particle physics and cosmology. It seems clear that ontological unity, in which objects and phenomena in particle physics and cosmology are interconnected, remains speculative. Even the more so, if the interconnection between cosmology and particle physics is conceived of in reductionist terms (i.e. if cosmology is imagined to be reducible to particle physics).

It could perhaps be objected that these hesitations are premature, for instance because the assumption of ontological unity between cosmology and particle physics has already lead to a number of interesting ideas which surely support the methodological impact of such a conjecture. Indeed, the problems discussed in this article might eventually provide fuel for new physics or new observations which could support the unity between cosmology and particle physics. Of course, nobody knows what the future will bring; but it should be noted that a methodological strategy of searching for unity - like other guiding principles - carries with it the danger of neglecting alternatives to programmes confined to this strategy. A worry along similar lines has been expressed by Burbidge:

...the beliefs developed over the last twenty years in this area [cosmology] have led to a total imbalance in the direction of research. [...] Far-out theoretical ideas are only taken seriously, if they are related to the very early universe. There, anything and everything goes. We are told that the unity between particle physics and astrophysics is upon us. Suppose there was no initial dense state? Suppose that matter and radiation were never strongly coupled together? Suppose that the laws of physics have evolved, as have everything else? The current climate of opinion requires that these questions not be asked. [5] 
One does not have to subscribe to Burbidge's alternative 'quasi steady-state' cosmology [6], or to his apparent sympathy for evolving laws of nature, to see that his questions are relevant to further critical philosophical analysis of the relationship between cosmology and particle physics.

On the other hand, if one takes seriously these challenges to unity between cosmology and particle physics, one might very well ask if I have anything to suggest instead. Without attempting a full analysis there seems to be at least two options. 1) Perhaps gravity is not a quantized force. As I have indicated, Bohr's anti-reductionist notion of unity, in which classical concepts are indispensable in quantum descriptions, is related to a more pessimistic view on the prospects of quantum gravity: If classical concepts (e.g. of time) are indeed necessary to discuss the early universe then it seems impossible to understand the universe of today solely as a consequence of a quantum gravity era, to the extent that this would be an attempt to understand the universe in terms of a 'quantum' reality. ${ }^{3}$ If the gravitational force is classical, it would mean that there is no quantum basis for understanding general relativity and thus no common unified framework for cosmology and particle physics. In this case, there can still be theoretical bridges between micro- and macro-physics, and the question of whether particle physics is relevant to cosmology is therefore left open. 2) Perhaps we should take a more critical approach to Big Bang; which, as Burbidge hints, is a central source of dreams of unity between cosmology and particle physics (given the supposed extreme energy conditions in the early universe). Of course, one might argue that even if the present understanding of the cosmological constant, inflation, dark matter etc. is all wrong, there is no need to question the overall Big Bang scenario. Nevertheless, if the standard picture is accepted until, say, $10^{-11} s$ after the Big Bang, it is hard to resist the temptation of extrapolating the Friedmann equation further back in time in search of the initial conditions of our universe. Thus, to really pursue this line of thinking, one would probably have to doubt some of the central pillars in the Big Bang theory.

Whatever the fate of these alternatives, it is fair to say that the connections between particle physics and cosmology, though often cherished, do not yet warrant a claim for a successful case of the unity of science. Again, this is not to say that the search for unity should not be pursued. It is just that the result should not be taken for granted.

\section{Acknowledgements}

I would like to thank Svend E. Rugh for many discussions related to the present article. It is also a pleasure to thank Jeremy Butterfield, Martín López Corredoira, Jan Faye, Helge Kragh, Mariano Moles, and Jesús Mosterín for comments on earlier drafts of this paper. Financial support from EU contract HPMF-CT-1999-00336 is gratefully acknowledged.

\footnotetext{
${ }^{37}$ Insofar as matter and radiation in the early Big Bang universe are assumed to be described exclusively in quantum (particle physics) terms, arguments of the in principle necessity of classical physics would also be relevant for a critical discussion of cosmology without quantum gravity.
} 


\section{References}

[1] Boerner, G. (1988) 'The present status of the inflationary universe' in Bertola, F. et al (eds) New Ideas in Astronomy (Cambridge: Cambridge University Press), pp. $275-278$

[2] Bohr, N. (1949) 'Discussion with Einstein on Epistemological Problems in Atomic Physics' in The Philosophical Writings of Niels Bohr - Vol.II, Essays 1932-1957 on Atomic physics and Human Knowledge, Reprint 1987, (Connecticut: Ox Bow Press) [originally, Wiley 1958], pp. 32-66

[3] Bosma, A. (1998) 'The Dark Matter Problem' (available at http://xxx.lanl.gov) astro-ph/9812015

[4] Brandenberger, R.H. (1999) 'Inflationary Cosmology: Progress and Problems' hepph/9910410

[5] Burbidge, G. (1988) 'Problems of cosmogony and cosmology' in Bertola, F. et al (eds) New Ideas in Astronomy (Cambridge: Cambridge University Press), pp. 223238

[6] Burbidge, G., Hoyle, F., and Narlikar, J.V. (1999) 'A Different Approach to Cosmology' April 1999 Physics Today, 38-46. See also Burbidge, G., Hoyle, F. and Narlikar, J.V. (2000) A Different Approach to Cosmology (Cambridge: Cambridge University Press)

[7] Butterfield, J. and Isham, C.J. (1999) 'On the Emergence of Time in Quantum Gravity' (gr-qc/9901024) in Butterfield, J. (ed) The Arguments of Time (Oxford: Oxford University Press), pp. 111-168

[8] Butterfield, J. and Isham, C.J. (2001) 'Spacetime and the Philosophical Challenge of Quantum Gravity' (gr-qc/9903072) in Callender, C. and Huggett, N. (eds) Physics Meets Philosophy at the Planck Scale (Cambridge: Cambridge University Press), pp. 33-89.

[9] Cat, J. (1998) 'The physicists' debates on unification in physics at the end of the 20th century' Historical Studies in the Physical and Biological Sciences 28, 253-299

[10] Dolgov, A.D. (1997) 'Baryogenesis, 30 years after' hep-ph/9707419

[11] Earman, J. and Mosterín, J. (1999) 'A Critical look at Inflationary Cosmology' Philosophy of Science 66, 1-49

[12] Einstein, A. (1918) 'Principles of research' reprinted in Einstein, A. Ideas and Opinions (New York: Dell Publishing Co. 1954), pp. 219-222

[13] Falkenburg, B. (1998) 'Bohr's Principles of Unifying Quantum Disunities' Philosophia naturalis 35 n.1, 95-120

[14] Faye, J. (1996) 'Moderne realistiske tolkninger af kvantemekanikken' [Modern realistic interpretations of quantum mechanics] Filosofiske Studier 16, 6-22

[15] Hacking, I. (1996) 'The Disunities of the Sciences' in Galison, P. and Stump, D.J. (eds.) The Disunity of Science - Boundaries, Contexts, and Power (Standford: Sandford University Press 1996) pp. 37-74

[16] Hartle, J.B. (1991) 'The Quantum Mechanics of Cosmology' in Coleman et al (eds.) Quantum Cosmology and Baby Universes (Singapore: World Scientific)

[17] Halliwell, J.J. (1993) 'Quantum Cosmology and the Creation of the Universe' in Hetherington, N.S. (ed) Cosmology: Historical, Literary, Philosophical, Religious, and Scientific Perspectives (New York: Garland Publ., Inc.), pp. 477-497

[18] Hendry, R.F. (1998) 'Quantum Mechanics, Experiment and Disunity - Comment 
on Peter Mittelstaedt' Philosophia naturalis 35 n.1, 153-159

[19] Hüttemann, A. (1998) 'Scientific Practice and the Disunity of Physics' Philosophia naturalis 35 n.1, 209-222

[20] Jaffe, A.H. et al (2000) 'Cosmology from Maxima-1, Boomerang and COBE/DMR CMB Observations' (astro-ph/0007333) Physical Review Letters 86, 3475-3479. See also e.g. Netterfield, C.B. et al (2001) 'A measurement by Boomerang of multiple peaks in the angular power spectrum of the cosmic microwave background' astro$\mathrm{ph} / 0104460$

[21] Kiefer C. (2000) 'Conceptual issues in quantum cosmology' gr-gc/9906100 Lecture Notes in Physics 541, 158-187

[22] Kilmister, C.W. (1994) Eddington's search for a fundamental theory (Cambridge: Cambridge University Press)

[23] Kolb, E.W. and Turner, M.S. (1993) The Early Universe (Reading: Addison-Wesley) (paperback edition)

[24] Kolb, E.W. (1994) 'Particle Physics and Cosmology', in K.J. Peach and L.L.J. Vick (eds.), High Energy Phenomenology (Fife: Univ. of St. Andrews Press), pp. 361-416

[25] Kragh, H. (1996) Cosmology and Controversy (Princeton: Princeton University Press)

[26] Liddle, A.R. (1999) 'Observational tests of inflation' astro-ph/9910110

[27] Linde, A.D. (1990) Particle Physics and Inflationary Cosmology (Chur: Harwood Academic Publishers)

[28] Lopez, R.E. and Turner, M.S. (1999) 'Precision Prediction for the Big Bang Abundance of Primordial He-4' (astro/ph-9807279) Physical Review D59, pp. 1035-1065

[29] Narlikar, J. (1987) The Primeval Universe (Oxford: Oxford University Press)

[30] North, J.D. (1965) The Measure of the Universe (Oxford: Oxford University Press)

[31] Peebles, P.J.E. (1999) 'Summary: Comments on the State of our Subject' astroph/9910234

[32] Ray, C. (1990) 'The Cosmological Constant: Einstein's greatest mistake?', Studies in History and Philosophy of Science 21 no.4, 589-604

[33] Rosenfeld, L. (1963) 'On quantization of fields' and Rosenfeld, L. (1966) 'Quantum Theory and Gravitation', both in Cohen, R.S. and Stachel, J. (eds.) Selected Papers of Léon Rosenfeld (Reidel: Dordrecht 1979), pp. 442-444 and 599-608

[34] Rovelli, C. (1999) 'Localization' in quantum field theory: how much of QFT is compatible with what we know about space-time' in Cao, T.Y. (ed) Conceptual Foundations of Quantum Field Theory (Cambridge: Cambridge University Press 1999), pp. 207-232

[35] Rugh, S. and Zinkernagel, H. (2000) 'The Quantum Vacuum and the Cosmological Constant Problem' hep-th/0012253

[36] Sakharov, A. (1967) JETP Letters 5, p. 24

[37] Sarkar, S. (1999) 'Big Bang Nucleosynthesis: Reprise' astro-ph/9903183

[38] Stachel, J. (1999) 'Introduction: Quantum field theory and space-time' and 'Comments' in Cao, T.Y. (ed) Conceptual Foundations of Quantum Field Theory (Cambridge: Cambridge University Press 1999), pp. 166-175 and 233-240

[39] Trodden, M. (1998) 'Electroweak Baryogenesis: A Brief Review' hep-ph/9805259

[40] Turner, M.S. and Tyson, J.A. (1999) 'Cosmology at the Millennium' (astro- 
ph/9901113) Review of Modern Physics 71, S145-S164

[41] Weinberg, S. (1989) 'The cosmological constant problem', Review of Modern Physics 61, 1-23

[42] Zinkernagel, P. (1962) Conditions for Description (New York: The Humanities Press)

[43] Zurek, W.H. (1991) 'Decoherence and the Transition from Quantum to Classical' Physics Today, October 1991, 36-44 